\title{
Neutrophil Extracellular Traps Open the Pandora's Box in Severe Malaria
}

\author{
Sebastian Boeltz ${ }^{1}$, Luis E. Muñoz ${ }^{1}$, Tobias A. Fuchs ${ }^{2}$ and Martin Herrmann ${ }^{1 *}$ \\ ${ }^{1}$ Department of Internal Medicine 3 - Rheumatology and Immunology, Friedrich-Alexander-University Erlangen-Nürnberg \\ (FAU) and Universitätsklinikum Erlangen, Erlangen, Germany, ${ }^{2}$ Institute of Clinical Chemistry and Laboratory Medicine, \\ University Medical Center Hamburg-Eppendorf, Hamburg, Germany
}

Keywords: severe malaria, cerebral malaria, neutrophil extracellular traps (NETs), Disseminated intravascular coagulation (DIC), vascular occlusion

\section{INTRODUCTION}

Malaria is transmitted by mosquitoes and kills 2,000 humans in sub-Saharan Africa each day (1). Most of the victims are children younger than 5 years of age. Out of the five plasmodium species causing malaria, Plasmodium falciparum is responsible for most of the severe and fatal infections (2). Upon feeding, the female Anopheles mosquito inoculates sporozoites that seek out the hosts' liver within minutes. After a first replication cycle in hepatocytes for 5-8 days, rupture releases the parasite into circulation where erythrocytes are infected. During the second replication cycle plasmodium consumes the contents and energy reserves of the erythrocytes, changes the membrane to enable adherence to the vessel walls (3), and produces waste, including crystalline urate (MSU) and hemozoin (4). After 24, 48, or $72 \mathrm{~h}$, depending on the species,

OPEN ACCESS

Edited by:

Janos G. Filep,

Université de Montréal, Canada

Reviewed by:

Stavros Giaglis,

University of Basel, Switzerland

Mohib Uddin,

AstraZeneca, Sweden

*Correspondence:

Martin Herrmann

martin.herrmann@uk-erlangen.de

Specialty section:

This article was submitted to Molecular Innate Immunity,

a section of the journal

Frontiers in Immunology

Received: 26 May 2017

Accepted: 10 July 2017

Published: 28 July 2017

Citation:

Boeltz S, Muñoz LE, Fuchs TA and Herrmann M (2017) Neutrophil

Extracellular Traps Open the Pandora's Box in Severe Malaria.

Front. Immunol. 8:874. doi: 10.3389/fimmu.2017.00874 the infected erythrocytes synchronously burst and release their content into the circulation and cause first clinical symptoms (Figure 1). The key pathogenic processes that cause severe malaria include rapid increase of infected erythrocytes, destruction of both infected and uninfected erythrocytes, acute inflammation, and microvascular obstruction. The final outcome is a reduced tissue perfusion that leads to downstream events compromising the cellular metabolism (5). We hypothesize that intravascular formation of neutrophil extracellular traps (NETs) contributes to the vasculopathy, driving severe malaria. NETs are web-like structures of highly modified chromatin and antimicrobial peptides released by activated neutrophils (6). In this article, we discuss the evidence supporting the role of NET formation in the pathogenesis of malaria and propose potential therapeutic interventions.

\section{NETS CAUSE VASCULOPATHY IN MALARIA}

NETosis has evolved as an important innate strategy for killing extracellular pathogens (7). The sticky chromatin fibers of NETs immobilize pathogens and inhibit their spread. Uric acid is to be found in infected erythrocytes as a precipitate together with high concentrations of hypoxanthine and the insoluble hemoglobin metabolite hemozoin. During rupture of infected erythrocytes, these compounds are abruptly released into the circulation. In this scenario, insoluble uric acid and hemozoin encounters the immune system as crystalline matter (4) and the former crystallizes in the sodium-rich plasma as MSU (8). These crystals are potent inducers of NETs formation, even in the presence of plasma proteins (8). Hemozoin activates leukocytes through an inflammasomedependent pathway (9). Indeed, it has been described that NETs with trapped parasites circulate in children infected with $P$. falciparum (10).

In flow chambers perfused with blood, NETs promote fibrin deposition and bind fibronectin and von Willebrand factor, important for platelet adhesion and thrombus propagation (11). NET fibers contain further procoagulant factors. Neutrophil elastase in NETs may proteolytically inactivate tissue factor pathway inhibitor (12). Furthermore, tissue factor can be deposited on extracellular 


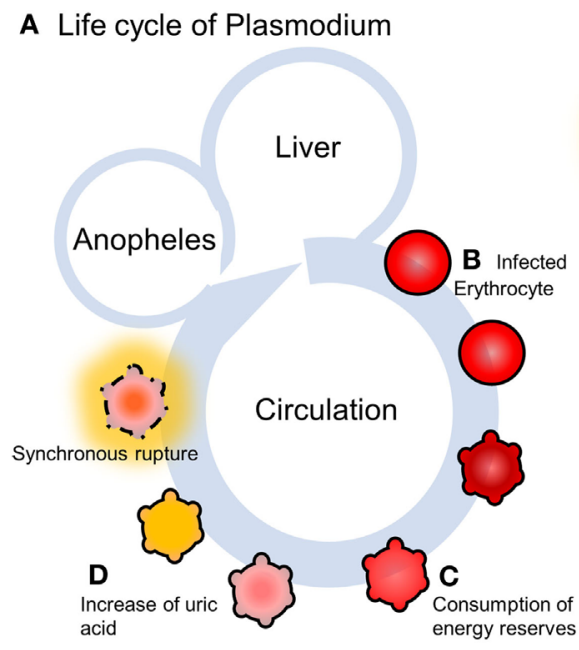

\section{E Rupture and synchronous release of Uric acid and Hemozoin}

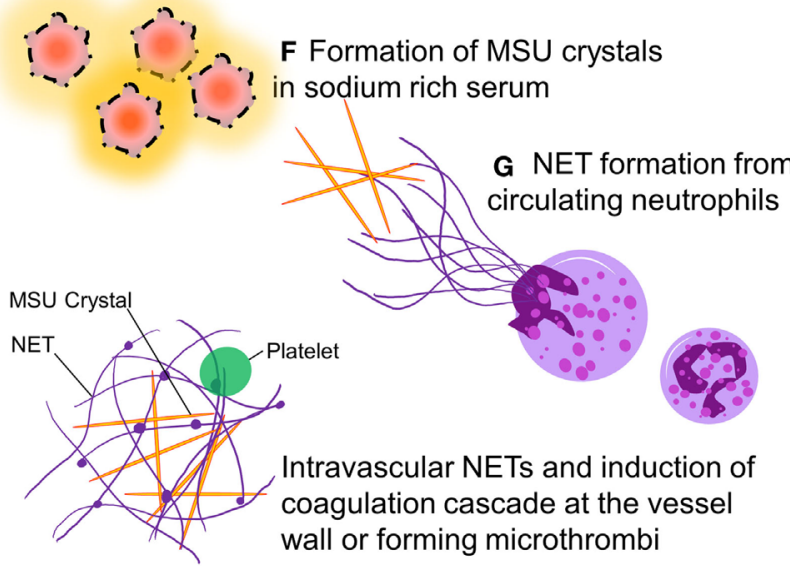

Microvasculature

(e.g. endothelium of postcapillary venules/ blood brain barrier)

$\begin{array}{lll}\text { H } & \text { Situation } & \text { Healthy } \\ \text { Endothelium } & \text { Thin, tight } \\ \text { Uric acid } & \text { low } \\ \text { Flow } & \text { normal }\end{array}$

I Situation Inflamed

Endothelium Thicker/ reduced function

Uric acid high

Flow reduced

J Situation NETs, inflamed, coagulation

Endothelium Thick/ disrupted/ loss of function

Uric acid Crystallization to MSU

Flow abrogated
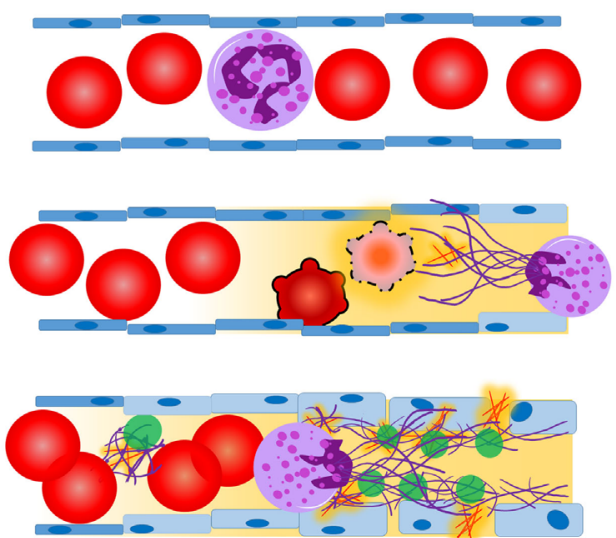

FIGURE 1 Hypothesis: intravascular neutrophil extracellular traps (NETs) precipitate severe malaria. Upon feeding, female Anopheles mosquitoes inoculate the host with sporozoites (A) which, within minutes, invade hepatocytes and replicate. A single sporozoite produces 10,000-30,000 merozoites within 5-8 days. After this the hepatocytes burst, merozoites enter the circulation, and invade erythrocytes and replicate (B). Depending on the species, the blood stage takes 24,48 , or $72 \mathrm{~h}$. During the growth within the erythrocytes, the parasite consumes the cells' cytoplasm and energy resources (C). Replicating parasites produce and store waste-like uric acid (orange) and hemozoin (D). During the blood stage, the parasite population increases 6-20 times per cycle. If the resources of the erythrocyte are consumed, rupture of the host erythrocyte releases the parasites and their waste including uric acid (E), which tends to form MSU crystals in the sodium-rich plasma with time (F). MSU crystals induce endothelial inflammation and NET formation upon contact with circulating neutrophils (G). In healthy conditions, the endothelium is a thin, monolayered, tight structure (H). Intravascular NET autonomously narrow or even clog the post-capillary venules, a process that can be accelerated by the activation of the coagulation cascade (I). The activation of the coagulation cascade, the recruitment of platelets in direct interplay with intravascular NETs, and a plethora of pro-inflammatory mediators cause microvascular occlusion and disrupt the blood-brain barrier, respectively (J).

traps, especially those derived from eosinophils, and factor XII is reportedly present and active on NETs (13). The negatively charged DNA in NETs may provide a scaffold for factor XII activation, which is aided by platelets, but the mechanism is still elusive. Fibrin and NETs synergize to augment the microbial defense in a process referred to as immunothrombosis (14). We hypothesize that the formation of NETs and the activation of the coagulation cascade is a double-edged sword. On the one side, they form a barrier on the endothelial surface protecting it from damage by MSU crystals (15) and hemozoin. On the other side, their occurrence limits the bore diameter of capillaries and postcapillary venules. This is prone to restrict perfusion of the end organs. In worst case, intravascular NETs autonomously clog the vessels or initiate disseminated intravascular coagulation (DIC), a condition involving hemorrhage and microthrombosis that synergize in the abolishment of tissue perfusion (Figure 1). The inflammatory mediators released during the coupled processes of NET formation and coagulation cause opening of the neuroimmunological blood-brain barrier and precipitate the clinical disease as, often fatal, cerebral malaria.

The clinical aspects of malaria vary with age, geography, epidemiology, and immunity. In endemic areas, young children and pregnant women are at highest risk to develop severe forms of malaria. Here, we want to interlace NET formation in plasmodium 
BOX 1 | Hypotheses for neutrophil extracellular traps (NETs) as pathognomonic factor for severe malaria.

1. Impaired consciousness: Intravascular NET formation and subsequent reduction of capillary bore diameters impair brain perfusion. In addition, toxic metabolites, e.g., ammonium, accumulate due to liver and kidney deficiency.

2. Prostration: Lack of perfusion of skeletal muscles due flow restriction in their capillaries. Flooding the patient with cytokines like IL1 $\beta$.

3. Multiple convulsions: NET-driven disseminated intravascular coagulation (DIC) and microthrombi cause multiple focal brain lesions and convulsions.

4. Acidosis: NET formation and acidosis are mutually influencing each other Reduced blood flow causes hypoxia and drop in the plasma $\mathrm{pH}$. However acidic conditions interfere with NET formation (17).

5. Hypoglycemia: Impaired perfusion and high consumption of glucose by Plasmodium deprives host tissues from glucose and precipitates end organ damage.

6. Renal impairment: Kidney is damaged due to hypoperfusion and glomerular obstruction. Reduced renal uric acid secretion causes hyperuricemia and promotes further NET formation.

7. Hemorrhages: NET-driven DIC contributes to the consumption of coagulation factors.

8. P. falciparum parasitemia ( $>10 \%)$ : Infected as well as ruptured erythrocytes are trapped by intravascular NETs, escaping sequestration in liver and spleen.

9. Severe malarial anemia, jaundice, pulmonary edema, and shock: There is no obvious specific contribution of NETs, besides the fact that these symptoms are the consequence of decreased end organ perfusion.

infections as key factor for the development of severe clinical manifestations (Box 1) $(2,16)$.

\section{DISCUSSION}

Each year, 240 million people develop symptomatic malaria infections, placing malaria as one of the most serious diseases of mankind. Treatment options in affected areas are challenging and casualties remain high. Here, we propose NET formation as key event determining the severity of disease progression. Parasites within erythrocytes reportedly produce uric acid, which is released as MSU crystals together with crystalline hemozoin synchronously upon rupture of the infected cells. MSU crystals may induce the formation of intravascular NETs, and hemozoin pigments activate neutrophils, immobilizing parasites and crystals at the endothelium. We discussed three possible outcomes (Figure 1). (i) The NETs shield the endothelium, dampening the pro-inflammatory effects of MSU (15) and hemozoin (9). (ii) The NETs and the activation of the coagulation cascade form a barrier on the surface of endothelium, reducing the vessels' inner diameter and, thereby, the perfusion to virtually all organs. (iii) NETs autonomously, as well as the activation of the coagulation cascade by the negatively charged DNA strands promote DIC. As consequence, hemorrhages and microthrombi may impact microcirculation and promote end organ ischemia. The interplay between intravascular NETs and the coagulation cascade is still elusive. However, the formation of a scaffold in the vasculature by NETs allows the recruitment of coagulation factors, inflammatory mediators, immune cells, and platelets-quite possible being able to disrupt the blood-brain barrier and promote cerebral malaria (18).

Intriguingly, reports indicate a decrease in the counts of circulating granulocytes, monocytes, and lymphocytes in the face of
Plasmodium infections, especially during gestation. This includes a significant reduction in peripheral neutrophil count (19). We speculate this might be partly due to the formation of NETs in the course of the disease. However, there is no direct evidence correlating the neutrophil count and the release of parasites into the circulation. An abnormal capacity to execute an oxidative burst was described for neutrophils of children suffering from $P$. falciparum malaria (20). The authors show that the reduced oxidative burst capacity in neutrophils occurred after the infection. In a murine model for malaria, this effect was attributed to the induction of the cytoprotective heme oxygenase 1 in neutrophil progenitors of the bone marrow (20). Although NET formation is closely associated with oxidative burst, the aforementioned study did not address NET formation. Therefore, we prompt researchers to include NET formation in their repertoire for functional evaluation of neutrophils. A number of studies argue that antimalarial drugs might influence the capacity of neutrophils to undergo oxidative burst or alter their effector functions $(21,22)$. Resulting limitation of neutrophils to form NETs might be beneficial in the context of malaria.

The occurrence of anti-neutrophil cytoplasmic antibodies (ANCAs) in $50 \%$ of patients with malaria supports the role of NETs in the etiopathogenesis of this, often fatal, disease $(23,24)$. The detection of these autoantibodies is used in the diagnosis of systemic vasculitides, e.g., granulomatosis with polyangiitis. ANCAs are classified into C- and P-ANCAs according to their antigen specificity for proteinase 3 and myeloperoxidase, respectively. While C- and P-ANCA bind to intact neutrophils, NETs contain only antigens of P-ANCAs (25). Malaria patients primarily develop P-ANCAs (23), supporting the notion that NETs may serve as an autoantigen in this disease.

The involvement of NETs in severe malaria has not been studied in detail. Utilizing NET formation, MSU, and the coagulation cascade as targets of new therapies harbors the potential to reduce the cases with mortality outcomes and alleviate severe forms of malaria infection.

\section{AUTHOR CONTRIBUTIONS}

SB wrote the manuscript. LM, MH, and TF supervised the project and wrote the manuscript. All the authors read and approved the manuscript.

\section{ACKNOWLEDGMENTS}

The authors acknowledge the support by Deutsche Forschungsgemeinschaft and Friedrich-Alexander-Universität Erlangen-Nürnberg (FAU) within the funding program Open Access Publishing.

\section{FUNDING}

This work was partially supported by the German Research Foundation (DFG) to MH (CRC1181-C03, KFO257, SPP 1468 Osteoimmunology IMMUNOBONE) and TF (FU741/4-1, INST 152/692) and by the EU H2020-MSCE-RISE-2015 project Nr. 690836 PANG and Volkswagen-Stiftung to MH and Arthea Biosciences, Inc. to LM. 


\section{REFERENCES}

1. White NJ, Pukrittayakamee S, Hien TT, Faiz MA, Mokuolu OA, Dondorp AM. Malaria. Lancet (2014) 383(9918):723-35. doi:10.1016/ S0140-6736(13)60024-0

2. WHO. Severe malaria. Trop Med Int Health (2014) 19(Suppl 1):7-131. doi:10.1111/tmi.12313_2

3. Tilley L, Dixon MW, Kirk K. The Plasmodium falciparum-infected red blood cell. Int J Biochem Cell Biol (2011) 43(6):839-42. doi:10.1016/j. biocel.2011.03.012

4. Gallego-Delgado J, Ty M, Orengo JM, van de Hoef D, Rodriguez A. A surprising role for uric acid: the inflammatory malaria response. Curr Rheumatol Rep (2014) 16(2):401. doi:10.1007/s11926-013-0401-8

5. Miller LH, Baruch DI, Marsh K, Doumbo OK. The pathogenic basis of malaria. Nature (2002) 415(6872):673-9. doi:10.1038/415673a

6. Remijsen Q, Vanden Berghe T, Wirawan E, Asselbergh B, Parthoens E, De Rycke R, et al. Neutrophil extracellular trap cell death requires both autophagy and superoxide generation. Cell Res (2011) 21(2):290-304. doi:10.1038/ cr. 2010.150

7. Brinkmann V, Reichard U, Goosmann C, Fauler B, Uhlemann Y, Weiss DS, et al. Neutrophil extracellular traps kill bacteria. Science (2004) 303(5663):1532-5. doi:10.1126/science. 1092385

8. Schorn C, Janko C, Krenn V, Zhao Y, Munoz LE, Schett G, et al. Bonding the foe - NETting neutrophils immobilize the pro-inflammatory monosodium urate crystals. Front Immunol (2012) 3:376. doi:10.3389/fimmu.2012.00376

9. Olivier M, Van Den Ham K, Shio MT, Kassa FA, Fougeray S. Malarial pigment hemozoin and the innate inflammatory response. Front Immunol (2014) 5:25. doi:10.3389/fimmu.2014.00025

10. Baker VS, Imade GE, Molta NB, Tawde P, Pam SD, Obadofin MO, et al. Cytokine-associated neutrophil extracellular traps and antinuclear antibodies in Plasmodium falciparum infected children under six years of age. Malar J (2008) 7:41. doi:10.1186/1475-2875-7-41

11. Fuchs TA, Brill A, Duerschmied D, Schatzberg D, Monestier M, Myers DD Jr., et al. Extracellular DNA traps promote thrombosis. Proc Natl Acad Sci U S A (2010) 107(36):15880-5. doi:10.1073/pnas.1005743107

12. Massberg S, Grahl L, von Bruehl ML, Manukyan D, Pfeiler S, Goosmann C, et al. Reciprocal coupling of coagulation and innate immunity via neutrophil serine proteases. Nat Med (2010) 16(8):887-96. doi:10.1038/nm.2184

13. von Bruhl ML, Stark K, Steinhart A, Chandraratne S, Konrad I, Lorenz M, et al. Monocytes, neutrophils, and platelets cooperate to initiate and propagate venous thrombosis in mice in vivo. J Exp Med (2012) 209(4):819-35. doi:10.1084/jem.20112322

14. Engelmann B, Massberg S. Thrombosis as an intravascular effector of innate immunity. Nat Rev Immunol (2013) 13(1):34-45. doi:10.1038/nri3345

15. Pieterse E, Jeremic I, Czegley C, Weidner D, Biermann MH, Veissi S, et al. Blood-borne phagocytes internalize urate microaggregates and prevent intravascular NETosis by urate crystals. Sci Rep (2016) 6:38229. doi:10.1038/ srep38229

16. Dondorp AM, Lee SJ, Faiz MA, Mishra S, Price R, Tjitra E, et al. The relationship between age and the manifestations of and mortality associated with severe malaria. Clin Infect Dis (2008) 47(2):151-7. doi:10.1086/589287

17. Maueroder C, Mahajan A, Paulus S, Gosswein S, Hahn J, Kienhofer D, et al. Ménage-à-trois: the ratio of bicarbonate to $\mathrm{CO}_{2}$ and the $\mathrm{pH}$ regulate the capacity of neutrophils to form NETs. Front Immunol (2016) 7:583. doi:10.3389/ fimmu.2016.00583

18. Schofield L, Grau GE. Immunological processes in malaria pathogenesis. Nat Rev Immunol (2005) 5(9):722-35. doi:10.1038/nri1686

19. Davison BB, Kaack MB, Rogers LB, Rasmussen KK, Rasmussen T, Henson EW, et al. Alterations in the profile of blood cell types during malaria in previously unexposed primigravid monkeys. J Infect Dis (2005) 191(11):1940-52. doi:10.1086/430004

20. Cunnington AJ, Njie M, Correa S, Takem EN, Riley EM, Walther M. Prolonged neutrophil dysfunction after Plasmodium falciparum malaria is related to hemolysis and heme oxygenase-1 induction. J Immunol (2012) 189(11):5336-46. doi:10.4049/jimmunol.1201028

21. Ferrante A, Rowan-Kelly B, Seow WK, Thong YH. Depression of human polymorphonuclear leucocyte function by anti-malarial drugs. Immunology (1986) 58(1):125-30.

22. Naisbitt DJ, Williams DP, O’Neill PM, Maggs JL, Willock DJ, Pirmohamed M, et al. Metabolism-dependent neutrophil cytotoxicity of amodiaquine: a comparison with pyronaridine and related antimalarial drugs. Chem Res Toxicol (1998) 11(12):1586-95. doi:10.1021/tx980148k

23. Pradhan V, Badakere SS, Shankarkumar U, Iyer YS, Ghosh K, Karnad D. Anti-neutrophil cytoplasmic antibodies (ANCA) in malaria. Indian J Malariol (2002) 39(3-4):51-9.

24. Yahya TM, Benedict S, Shalabi A, Bayoumi R. Anti-neutrophil cytoplasmic antibody (ANCA) in malaria is directed against cathepsin G. Clin Exp Immunol (1997) 110(1):41-4. doi:10.1111/j.1365-2249.1997.498-ce1395.x

25. Panda R, Krieger T, Hopf L, Renne T, Haag F, Rober N, et al. Neutrophil extracellular traps contain selected antigens of anti-neutrophil cytoplasmic antibodies. Front Immunol (2017) 8:439. doi:10.3389/fimmu.2017.00439

Conflict of Interest Statement: The authors declare that the research was conducted in the absence of any commercial or financial relationships that could be construed as a potential conflict of interest.

Copyright (c) 2017 Boeltz, Muñoz, Fuchs and Herrmann. This is an open-access article distributed under the terms of the Creative Commons Attribution License (CC BY). The use, distribution or reproduction in other forums is permitted, provided the original author(s) or licensor are credited and that the original publication in this journal is cited, in accordance with accepted academic practice. No use, distribution or reproduction is permitted which does not comply with these terms. 